\title{
La relación de intercambio entre explorar y explotar recursos internacionalmente: posibles recetas para el crecimiento internacional a corto plazo
}

\author{
Carina Silva $^{\mathrm{a}}$, Miguel González-Loureiro, ${ }^{1, \mathrm{~b}, \mathrm{a}}$, Vitor Braga ${ }^{\mathrm{a}}$ \\ ${ }^{a}$ CIICESI, ESTG, Instituto Politécnico de Porto, Portugal \\ ${ }^{b}$ Universidade de Vigo, España
}

doi: $10.20420 /$ eni.2020.366

\begin{abstract}
Resumen
Las PYMEs requieren actualmente un crecimiento rápido en mercados internacionales y una toma de decisiones racional en función del contexto. En este marco, estudiamos dos alternativas complementarias pero excluyentes -dedicar recursos a la exploración y a la explotación internacional- bajo un enfoque cognitivo, y su asociación con el crecimiento de las ventas internacionales en el corto plazo. Nuestro estudio revela que una mayor orientación a la explotación internacional se asocia positivamente con ese crecimiento. En cambio, una mayor orientación a la exploración internacional se asocia negativamente. Ofrecemos algunas recomendaciones para acelerar el crecimiento internacional basado en estos resultados.
\end{abstract}

Palabras clave: crecimiento internacional, ambidestría internacional, cognición del directivo.

Clasificación JEL: F20, F14.

Agradecimiento: Este trabajo ha sido financiado por fondos nacionales de investigación recibidos de la FCT - Fundação para a Ciência e Tecnologia de Portugal a través del proyecto UIDB/04728/2020. Miguel González-Loureiro también agradece los fondos recibidos del Ministerio de Ciencia e Innovación de España a través del proyecto PID2019-106677GB-I00.

Fuente de referencia: DOI: 10.4018/JGIM.2021010104. Silva, C., González-Loureiro, M., \& Braga, V. L. (2021). The Influence of Organizational Ambidexterity on SME Speed of Internationalization. Journal of Global Information Management, 29(1), 68-84.

\section{Introducción}

Las nuevas empresas internacionales (NEI), aquellas que se internacionalizan durante los seis primeros años desde su fundación, tienen la necesidad imperiosa de conseguir un retorno suficiente rápidamente tras su primera entrada al mercado internacional (Hennart, 2014). Esto es así porque se enfrentan simultáneamente a dos desventajas. Una, el hecho de ser nueva empresa con poca experiencia en la organización interna de la empresa -rutinas organizativas, precisión en las cuentas previsionales, gestión de cambios en la demanda y producción...-. Otra, el hecho de entrar por primera vez a un mercado internacional y su inexperiencia en la participación en estas redes de comercio internacional.

Hasta la fecha, se ha observado que estas NEI, que suelen ser pequeñas y medianas empresas-PYMEs, siguen estrategias de nicho de mercado (Hennart,
2014). Esto es, como se focalizan en atender sólo una parte de los grupos de clientes existentes en un mercado dado, deben ampliar el ámbito geográfico de mercado para mejorar su viabilidad económica y sobrevivir en el corto plazo. Son PYMEs que parecen nacidas para correr, puesto que necesitan extender rápidamente sus mercados geográficos en poco tiempo (Puig y otros, 2018).

Caben dos orientaciones a la hora de invertir los limitados recursos que estas PYMEs tienen en actividades internacionales. Una sería la orientación explotadora, que implica la inversión de recursos en rutinas organizativas actuales $y$ en el aprovechamiento comercial de los productos/mercados actuales. Otra sería la orientación exploradora, que supone la inversión de recursos en el descubrimiento y desarrollo de nuevas oportunidades de negocio en nuevos mercados internacionales, nuevas rutinas organizativas, o nuevos productos

${ }^{1}$ Autor de correspondencia: mloureiro@uvigo.es 
(Hsu y otros, 2013). Dicho de otro modo, la orientación explotadora equivale a invertir en ser competitivos hoy, y la exploradora a prepararse para seguir siendo competitivos en el futuro (Raisch \& Birkinschaw, 2008). Así, las empresas deben dedicar recursos a ambos tipos de actividades para asegurar el proyecto empresarial a largo plazo, en lo que se denomina ambidestría organizativa (Lubatkin y otros, 2006). Esto tiene potencial para ser traducido a su vez a la ambidestría internacional. Esto es, la capacidad de la organización para, simultáneamente, ser eficiente tanto en la gestión de la demanda de su negocio actual en mercados internacionales -explotación internacional- como en la adaptación a los cambios del entorno para competir en mercados internacionales en el futuro-exploración internacional.

No obstante, se produce una relación de intercambio entre la explotación y la exploración. Ante una cantidad de recursos dada, se ha de dedicar una proporción a explotar y otra a explorar, y no es posible aumentar la proporción dedicada a ambas, ya que la suma total debe ser el $100 \%$ del total de recursos disponibles. En el ámbito de la internacionalización de las PYMEs nos llevaría a la cuestión de cuál de las dos orientaciones, explotadora y exploradora, ayuda más a crecer internacionalmente.

Puesto que las decisiones en internacionalización las toma el equipo directivo basado en la representación mental del entorno y el negocio (Maitland \& Sammartino, 2015), lo anteriormente expuesto lleva a plantearse también la cuestión de en qué medida la cognición directiva puede explicar esa ambidestría internacional. La cognición directiva se basa en el uso que los directivos realizan de sus dos sistemas cognitivos, el sistema $\mathrm{C}$-analítico, que requiere uso de recursos cognitivos, consciente, deliberado- y el sistema $\mathrm{X}$-inmediato, sin esfuerzo cognitivo, intuitivo-(Kahneman, 2003). Este sistema intuitivo en nuestro estudio debe ser entendido como la intuición en negocios internacionales, la cual se fundamenta en el aprendizaje basado en la experiencia.

Dado que la experiencia es clave para explicar el proceso de internacionalización de las empresas en el denominado modelo de Uppsala o internacionalización gradual, (Johanson \& Vahlne,
1977, 2009) hemos realizado una investigación en empresas con relevantes necesidades de internacionalización bajo dicho enfoque gradual. Así, escogimos una muestra de empresas de sectores manufactureros tradicionales (textil, calzado, mobiliario) de Portugal, región ultra-periférica de Europa, cuyos resultados más detallados pueden consultarse en Silva y otros (2021). Ante el limitado tamaño de la demanda interna en Portugal, y ante su situación geográfica en un extremo de Europa mercado internacional más frecuente en las primeras internacionalizaciones-, estas empresas tienen la imperiosa necesidad de crecer rápidamente en el mercado internacional mediante estrategias de nicho de mercado. Nuestros hallazgos son, por tanto, extrapolables a empresas en contextos y situaciones similares.

Nuestra propuesta es que existe una relación de intercambio entre las orientaciones internacionales explotadora y exploradora que dificulta la ambidestría internacional lo que, a su vez, limita el crecimiento de las ventas internacionales en el corto plazo -inferior a 3 años. A su vez, el modo en el que los directivos utilizan ambos sistemas cognitivos -analítico e intuitivo- se asociará de forma diferente con la orientación internacional explotadora y exploradora de la PYME.

\section{Orientación explotadora vs. orientación exploradora: relación con el crecimiento internacional de las ventas}

La principal teoría que explica el comportamiento internacional de las empresas es el denominado modelo de internacionalización gradual o modelo de Uppsala. Esencialmente, todas las empresas son adversas al riesgo, aunque unas aceptan mayores niveles que otras. Invertir recursos en el primer mercado internacional implica la decisión de qué cantidad invertir, bajo qué modalidad de entrada (exportación indirecta, directa, subsidiaria de ventas en el exterior...) y en qué mercado. En esta primera entrada y en una situación en que ni la empresa ni el equipo directivo cuentan con experiencia internacional, la empresa preferirá una entrada que implique un bajo compromiso de recursos, lo cual implica menor riesgo. Así, la exportación indirecta o directa requiere menos recursos que una subsidiaria en el exterior y es también menos arriesgada. A medida que la empresa va adquiriendo el 
conocimiento basado en la experiencia en ese mercado y en redes de comercio internacional, entonces va sintiéndose más segura para ir comprometiendo cada vez más recursos en actividades internacionales y podrá pasar a modos de mayor compromiso. $\mathrm{Y}$ esto lleva a la perspectiva gradual en el proceso internacionalizador.

No obstante, se ha observado la existencia de nuevas empresas que, sin apenas tener algo de esa experiencia internacional, dan pasos de alto compromiso internacional desde el principio. Se ha demostrado que, esencialmente, se debe a la conjunción de orientación emprendedora del equipo directivo, tipo de industria en la que compite la empresa y a su estrategia de nicho de mercado (Hennart, 2014).

Ante esto, una orientación internacional ambidiestra debería de poder ser el acelerante del crecimiento de ventas en mercados internacionales (Prange \& Verdier, 2011). Esto es, la capacidad de la organización para simultáneamente vender internacionalmente a través de la explotación de recursos actuales y de la exploración de nuevos recursos.

Sin embargo, debemos tener en cuenta la existencia de un cierto retraso entre el momento de inversión de recursos y su retorno. Así, es de esperar que la explotación de recursos en mercados internacionales arroje resultados más tempranamente que la exploración. Esto se debe a que la exploración internacional requiere un tiempo entre la detección de oportunidades, el desarrollo de productos/servicios y la puesta en marcha de ese nuevo binomio producto/mercado (Dasí y otros, 2015). En cambio, la explotación internacional implica un menor tiempo de desarrollo, puesto que los productos/servicios ya existen, así como también las rutinas organizativas que lo soportan.

Se debe destacar la importancia del crecimiento en el corto plazo, puesto que las nuevas empresas que se internacionalizan tempranamente requieren un nivel determinado de crecimiento de las ventas internacionales para ser viables en el corto plazo (Puig y otros, 2018).

En nuestra muestra de empresas analizada, se observó precisamente esto. Una mayor orientación a explorar internacionalmente estaba asociada negativamente con el crecimiento de las ventas internacionales respecto al total de ventas en el corto plazo. Además, una mayor orientación a explotar internacionalmente estaba asociada positivamente con dicho crecimiento. Como en nuestro estudio contemplamos ambas orientaciones por separado, pudimos observar que la asociación era opuesta en signo. En cambio, si hubiésemos integrado ambas orientaciones en la denominada ambidestría organizativa como una única variable, entonces el efecto podría no haber sido significativo, puesto que uno compensaría al otro.

Destacamos que este hallazgo es contrario a lo esperado a nivel teórico, ya que parecería que las empresas que persiguen la exploración internacional crecerían más a nivel internacional que las que persiguen la explotación internacional (Prange \& Verdier, 2011). Al menos en el corto plazo, esto no es así. Y no lo es por el tiempo que debe transcurrir entre la inversión en esfuerzos explorativos y el momento en el que dichos esfuerzos empiezan a dar ventas internacionales.

\section{Aspectos cognitivos del directivo en su relación con ambidestría organizativa internacional y crecimiento de ventas internacionales}

La ambidestría internacional requiere tomar decisiones entre invertir en explotación o en exploración internacional. El uso del sistema cognitivo intuitivo, que es la intuición basada en la experiencia, sólo es confiable en un contexto en el cual se ha tenido tiempo de aprender que una serie de señales del entorno llevan a una determinada decisión, y que esa decisión fue correcta (Kahneman, 2003). Esto guarda relación con el mecanismo de la internacionalización gradual, puesto que la experiencia en mercados internacionales explica pasar de una etapa a otra en la internacionalización gradual. Por ello, no parece aconsejable que se confíe en ese sistema cognitivo intuitivo en decisiones de exploración internacional, puesto que implica nuevas rutinas, nuevos mercados, nuevos productos, o nuevos recursos en el ámbito de la internacionalización. Uno no puede fiarse de la intuición que tiene sobre negocios internacionales cuando dicha intuición fue adquirida en el mercado doméstico, y que luego trata de aplicarlo en nuevo contexto que solo puede 
asegurar que asemeja ser parecido a un contexto doméstico o internacional anterior $\sin$ antes asegurarse de que efectivamente lo es (Vlačić y otros, 2021). Por ello, el sistema cognitivo analítico parece el más adecuado y confiable para tomar decisiones sobre exploración internacional. Solamente a través de un análisis deliberado se puede llegar a conclusiones confiables en la decisión de exploración internacional.

Por otro lado, las decisiones de dedicar recursos a la explotación internacional implican profundizar en mayor medida en mercados internacionales en los que ya se está, en los que ya se tiene experiencia, con productos/servicios actuales y con las rutinas organizativas y recursos actuales. Ha habido tiempo para aprender en base a la experiencia en este tipo de actividades. Por tanto, tiene sentido usar la intuición basada en la experiencia. Adicionalmente, la intuición basada en la experiencia guiará al sistema cognitivo analítico en la elección de qué datos y hechos hay que analizar (Vlačić y otros, 2021), porque el decisor se enfrenta a una tarea similar a lo ya realizado anteriormente. El sistema analítico debe usarse para evaluar si la intuición que se tiene sobre la explotación internacional es correcta, para aportar información complementaria que no es posible adquirir mediante la experiencia, sino mediante el análisis de datos y hechos. Al basarse en la experiencia adquirida en esos mismos mercados internacionales, la combinación de ambos sistemas cognitivos dará lugar a una mayor orientación explotadora internacional.

Nuestros resultados corroboraron estas ideas. Existe una asociación positiva entre el uso de ambos sistemas cognitivos de los directivos y una mayor orientación explotadora internacionalmente. En cambio, solamente hay una asociación positiva entre el sistema cognitivo analítico y una mayor orientación exploradora internacional. Adicionalmente, no hay una relación directa de ambos sistemas cognitivos y el crecimiento de la proporción de ventas internacionales respecto al total de ventas.

\section{Conclusiones: posibles recetas para acelerar el crecimiento internacional en el corto plazo}

Los hallazgos anteriores permiten concluir que para acelerar el crecimiento a corto plazo de las ventas internacionales es necesario dedicar más recursos a la explotación que a la exploración internacionales. Además, los directivos de empresas que deseen crecer rápidamente en mercados internacionales deben utilizar ambos sistemas cognitivos y pueden tanto cimentarse en su intuición basada en la experiencia como en análisis detallados. Sin embargo, no deben confiar en su intuición para tomar decisiones sobre exploración internacional.

Si se dedican más recursos a explorar que a explotar, la asociación negativa de la exploración internacional y la proporción de ventas internacionales hará peligrar el crecimiento a corto plazo, lo que podría llevar al proyecto empresarial al fracaso. No obstante, esto no significa que haya que dejar de lado la exploración. La exploración internacional ha de tener su horizonte temporal a un plazo superior a tres años y, además, no sería posible continuar creciendo de forma sostenida en el tiempo si no se dedican recursos a explorar.

No obstante, los directivos de empresas con necesidad de crecer internacionalmente deben tener en cuenta que los recursos dedicados a explotar mercados internacionales son detraídos de aquellos dedicados a explorar nuevos mercados internacionales. Por tanto, se produce en el corto plazo una tensión, un difícil equilibrio entre dedicar recursos a ser competitivo hoy o a prepararse para ser competitivos en el futuro. No hay futuro si no se consigue un crecimiento determinado en el corto plazo, pero tampoco se puede asegurar el crecimiento futuro sólo dedicando recursos a explotar los mercados internacionales que se tengan en un momento dado.

Como estas empresas desarrollan estrategias de nicho y la demanda interna es limitada, deben crecer rápidamente en mercados internacionales. Un crecimiento rápido en el corto plazo mediante la explotación internacional facilitará obtener recursos hoy que luego se pueden invertir en el medio plazo para seguir creciendo mañana -exploración. Esto es, se estaría aumentando la cantidad total de recursos futuros a repartir entre explotación y exploración.

Los directivos además deben fiarse más de un sistema cognitivo que de otro, dependiendo de si lo que quieren es tomar decisiones de inversión en actividades internacionales explotadoras o exploradoras. La intuición basada en la experiencia 
solamente es confiable y se asocia positivamente con una mayor orientación a la explotación internacional. En cambio, tanto el razonamiento analítico como la intuición en los negocios se asocia positivamente con mayor orientación explotadora.

Queda la paradoja de la primera internacionalización. Ante esa situación, estaríamos ante una actividad eminentemente exploradora, puesto que es un nuevo mercado y requerirá cambios organizativos que también son exploradores. Sin embargo, si la exploración se asocia negativamente con el crecimiento de las ventas internacionales, ¿cómo es posible explicar el crecimiento derivado de esa primera entrada exploradora?

En base a nuestros resultados, si el equipo directivo plantea esto como puramente explorador, puede tener resultados negativos en el corto plazo. Esa primera internacionalización, que tiene un carácter inherentemente explorador, ha de combinar explotación y exploración. Esto es, se debe buscar poca o ninguna adaptación en el producto actual (explotación), buscar la ayuda de agentes con experiencia en mercados internacionales -por tanto estaríamos transformando nuestra exploración en explotación por parte de un agente con experiencia en ese producto/mercado-, o contratar personal con experiencia para suplir la carencia inicial de experiencia organizativa, y tratar así de reducir al máximo los niveles de exploración en el corto plazo que se asociarán con un crecimiento de ventas menor. $\mathrm{Y}$, por supuesto, recomendaríamos al equipo directivo que no tratase de extrapolar su intuición en los negocios extraída de su experiencia en el mercado doméstico a lo que parece un mercado internacional similar. Sin tener la total seguridad de que efectivamente ese primer mercado internacional es similar al doméstico, se debe usar el razonamiento analítico, el cual además está menos expuesto a los denominados sesgos cognitivos que llevan a decisiones erróneas ante contextos nuevos.

\section{Referencias}

Dasí, A., Iborra, M., \& Safón, V. (2015). Beyond path dependence: Explorative orientation, slack resources, and managerial intentionality to internationalize in SMEs. International Business Review, 24(1), 77-88.
Hennart, J. F. (2014). The accidental internationalists: a theory of born globals. Entrepreneurship Theory and Practice, 38(1), 117-135.

Hsu, C. W., Lien, Y. C., \& Chen, H. (2013). International ambidexterity and firm performance in small emerging economies. Journal of World Business, 48(1), 58-67.

Johanson, J., \& Vahlne, J.-E. (1977). The internationalization process of the firm-a model of knowledge foreign and increasing market commitments. Journal of International Business Studies, 8(1), 23-32.

Johanson, J., \& Vahlne, J. E. (2009). The Uppsala internationalization process model revisited: From liability of foreignness to liability of outsidership. Journal of International Business Studies, 40(9), 1411-1431.

Kahneman, D. (2003). A perspective on judgment and choice: Mapping bounded rationality. American Psychologist, 58(9), 697-720.

Lubatkin, M. H., Simsek, Z., Ling, Y., \& Veiga, J. F. (2006). Ambidexterity and performance in small-to medium-sized firms: The pivotal role of top management team behavioral integration. Journal of Management, 32(5), 646-672.

Maitland, E., \& Sammartino, A. (2015). Managerial cognition and internationalization. Journal of International Business Studies, 46(7), 733-760.

Prange, C., \& Verdier, S. (2011). Dynamic capabilities, internationalization processes and performance. Journal of World Business, 46(1), 126133.

Puig, F., Gonzalez-Loureiro, M., \& Ghauri, P. N. (2018). Running faster and jumping higher? Survival and growth in international manufacturing new ventures. International Small Business Journal: Researching Entrepreneurship, 36(7), 829-850.

Raisch, S., \& Birkinshaw, J. (2008). Organizational ambidexterity: Antecedents, outcomes, and moderators. Journal of Management, 34(3), 375-409

Silva, C., González-Loureiro, M., \& Braga, V. L. (2021). The influence of organizational ambidexterity on SME speed of internationalization. Journal of Global Information Management, 29(1), 68-84.

Vlačić, B., González-Loureiro, M., \& Eduardsen, J. (2021). The process of the process of internationalisation: cognitive and behavioural perspectives in small ventures. European Journal of International Management, avance on line, DOI: 10.1504/EJIM.2020.10027574. 